\title{
Discrete and intersample analysis of systems with aperiodic sampling
}

\author{
L. Hetel, A. Kruszewski, W. Perruquetti, J.P. Richard
}

\begin{abstract}
This article addresses the stability analysis of linear time invariant systems with aperiodic sampleddata control. Adopting a difference inclusion formalism, we show that necessary and sufficient stability conditions are given by the existence of discrete-time quasi-quadratic Lyapunov functions. A constructive method for computing such functions is provided from the approximation of the necessary and sufficient conditions. In practice, this leads to sufficient stability criteria under LMI form. The inter-sampling behavior is discussed there: based on differential inclusions, we provide continuous-time methods that use the advantages of the discrete-time approach. The results are illustrated by numerical examples that indicate the improvement with regard to the existing literature.
\end{abstract}

\section{Index Terms}

aperiodic sampled-data control, difference inclusions, stability, quasi-quadratic functions.

\section{INTRODUCTION}

The stability analysis of linear time invariant (LTI) systems with aperiodic sampling is a very challenging question. This problem is not easy, since, under variations of the sampling interval, the trajectory of a system may become unstable (see [23], page 69). The problem is relevant to networked / embedded control applications and has been addressed from both the discrete-time and continuous-time points of view.

In continuous-time, it has been approached using a time-delay system modeling [7], [6], a norm-bounded uncertainty modeling of the sample-and-hold operator [18], [9] or an impulsive

The authors are with CNRS LAGIS, FRE 3303, Ecole Centrale de Lille, BP48, 59651 Villeneuve d'Ascq Cedex, France.

W. Perruquetti and J.P. Richard are also involved in the INRIA, Projet ALIEN, France.

Corresponding author: laurentiu.heteleec-lille.fr 
system model [20]. The disadvantage of continuous-time methods is that, in general, the analysis does not take into account the particular variation of the sampling-induced delay which exhibits a "sawtooth shape". The only method that considers this issue is the recent work [6]. Continuoustime methods may also suffer from conservatism due to the upper-bounding of the derivatives of Lyapunov-Krasovskii functionals or to the symmetry of ellipsoidal norms used for bounding the sample-and-hold operator.

In the discrete-time domain, using the exact integration over a sampling interval, the system with aperiodic sampling can be expressed as a linear difference inclusion (LDI): see [21], [5] for a switched LDI, [13] for a polytopic LDI, [1], [8] for a norm-bounded LDI and [22], [19], [14], [4] for generic stability properties of LDI. Sufficient linear matrix inequality (LMI) conditions for stability are given in the literature, based on the existence of quadratic Lyapunov functions [21], [5], [8], or of Lyapunov functions that depend on the sampling interval [13], [3]. Compared to continuous-time approaches, discrete-time methods profit by involving an integration procedure that implicitly takes into account the particular nature of the sampling-induced delay. The main drawback is that they cannot take into account the intersample system behavior. Besides, they become numerically inaccurate when the minimum sampling interval tends to zero.

This paper concerns both discrete-time (Section III) and continuous-time (Section IV) approaches for the stability analysis of LTI systems under aperiodic sampling. The LDI model suggests that improvement in the stability analysis can be made by using quasi-quadratic Lyapunov functions [19], [14], [24] which are both necessary and sufficient for the stability of LDI. However, the computation of such functions is still an open problem. To the authors knowledge, there is no LMI criteria in the literature for deriving such functions. This paper provides such a constructive numerical procedure for deriving quasi-quadratic Lyapunov functions. The approach is based on a necessary and sufficient condition that underlines a converse Lyapunov theorem for the stability of LDI defined on compact sets (as the LDI obtained from systems with aperiodic sampling). In practice, some restrictions to sufficient conditions lead to a LMI characterization of the stability domain that can be favorable compared to the existing literature. It is possible to tune the amount of conservatism of these LMI according to the desired numerical complexity. In a second time, we analyze systems with aperiodic sampling from the continuous-time point of view. The goal is to propose a new approach that uses the advantages of the discrete-time methods, via the classical integration operator. The approach can be used for analyzing the 
intersample system behavior.

This paper is organized as follows: Section II provides a mathematical formulation of the problem under study; Section III deals with the discrete-time analysis, while Section IV presents continuous-time results. Numerical examples are presented in Section V.

Notations : For a square symmetric matrix, $M \succ 0(M \prec 0)$ indicates that $M$ is positive (negative) definite. $\|\cdot\|_{M}$ denotes the ellipsoidal norm associated to a matrix $M=M^{T} \succ 0$, $\|x\|_{M}=\sqrt{x^{T} M x}$. By $\lambda_{\max }(M)\left(\lambda_{\min }(M)\right)$ we denote the maximum (minimum) eigenvalue of a square symmetric matrix $M$. For a given set $\mathcal{F}$, the symbol $c o \mathcal{F}$ denotes the convex hull of the set. For $p \in \mathbb{N}, \mathcal{I}_{p}$ denotes the set $\{1,2, \ldots, p.\} \subset \mathbb{N}$. For $k \in \mathbb{N}$ and a compact set $\Theta$ we denote by $S_{k}(\Theta)=\left\{\sigma: \sigma=\left\{\theta_{i}\right\}_{i=0}^{k-1}, \theta_{i} \in \Theta, \forall i=0, \ldots, k-1\right\}$ the set of all $k$ - length sequences with values in $\Theta$. By $\nabla_{y} V(x):=\lim _{\epsilon \rightarrow 0^{+}}(V(x+\epsilon y)-V(x)) \epsilon^{-1}$ we denote the directional derivative of a function $V(x)$ along the direction $y$.

\section{MATHEMATICAL FORMULATION}

Consider two positive integers $n, m$ and the matrices $A_{c} \in \mathbb{R}^{n \times n}, B_{c} \in \mathbb{R}^{n \times m}$. We are interested in the class of LTI systems $\dot{x}(t)=A_{c} x(t)+B_{c} u(t), \forall t \in \mathbb{R}^{+}, x(t)=x_{0} \in \mathbb{R}^{n}, \forall t \leq 0$. Here $x: \mathbb{R} \rightarrow \mathbb{R}^{n}$ represents the system state and $u: \mathbb{R} \rightarrow \mathbb{R}^{m}$ represents the control. We consider that the control law is a piecewise constant state feedback, i.e. $u(t)=K x\left(t_{k}\right), \forall t \in\left[t_{k}, t_{k+1}\right)$, where $\left\{t_{k}\right\}_{k \in \mathbb{N}}$ represents an unbounded monotonously increasing sequence of sampling instants with elements in $\mathbb{R}^{+}$, i.e.

$$
0=t_{0}<t_{1}<\ldots<t_{k}<\ldots ; t_{k} \in \mathbb{R}^{+}, \forall k \in \mathbb{N} ; \lim _{k \rightarrow \infty} t_{k}=\infty
$$

We denote by $\theta_{k}:=t_{k+1}-t_{k}$ the sampling interval and we consider that for all $k, \theta_{k}$ belongs to a compact set $\mathcal{T} \subset \mathbb{R}^{+}$. The closed-loop system has the form

$$
\dot{x}(t)=A_{c} x(t)+B_{c} K x\left(t_{k}\right), \forall t \in\left[t_{k}, t_{k+1}\right), x(t)=x_{0} \in \mathbb{R}^{n}, \forall t \leq 0 .
$$

We denote by $\theta_{\min }$ and $\theta_{\max }$ the minimum and maximum of $\mathcal{T}$, respectively, $0<\theta_{\min }<$ $\theta_{\max }<\infty$. The problem under study is formulated as follows:

Problem : Is system (2) stable for all the possible sampling sequences satisfying $\theta_{k} \in \mathcal{T}$ and assumption (1) ? 
The study in this paper will be grounded on the use of the integration operator $\Lambda: \mathbb{R}^{+} \rightarrow \mathbb{R}^{n \times n}$,

$$
\Lambda(\theta):=I+\int_{0}^{\theta} e^{s A_{c}} d s\left(A_{c}+B_{c} K\right) .
$$

The operator $\Lambda(\cdot)$ is continuous w.r.t. $\theta$, and $\theta$ belongs to the compact set $\mathcal{T}$. Then the set $\{\Lambda(\theta): \theta \in \mathcal{T}\}$ is a compact subset of $\mathbb{R}^{n \times n}$. We consider that there exists a polytopic set

$$
\mathcal{Z}=\operatorname{co}\left\{Z_{1}, Z_{2}, \ldots, Z_{p}\right\} \subset \mathbb{R}^{n \times n}
$$

with a finite number $p$ of vertices, so that $\Lambda(\theta) \in \mathcal{Z}, \forall \theta \in \mathcal{T}$. Similarly, we consider the set

$$
\mathcal{W}=\operatorname{co}\left\{W_{1}, W_{2}, \ldots, W_{p}\right\} \subset \mathbb{R}^{n \times n}
$$

with a finite number $p$ of vertices, so that $\Lambda(\theta) \in \mathcal{W}, \forall \theta \in\left[0, \theta_{\max }\right]$. Methods for the construction of such polytopic sets exist in the literature [12], [13], [11] and will not be discussed in this paper. In what follows, we use the integration operator and the convex sets (4), (5) for both a discrete-time and continuous-time analysis of systems with aperiodic sampling.

\section{DISCRETE-TIME ANALYSIS}

\section{A. Generalities}

In this section we recall some basic stability properties and the construction of a LDI model for systems with aperiodic sampling. For all $t \in\left[t_{k}, t_{k+1}\right]$ the solutions of system (2) satisfy:

$$
\begin{aligned}
x(t) & =e^{\left(t-t_{k}\right) A_{c}} x\left(t_{k}\right)+\int_{0}^{\left(t-t_{k}\right)} e^{s A_{c}} d s B_{c} K x\left(t_{k}\right) \\
& =\left(I+\int_{0}^{\left(t-t_{k}\right)} e^{s A_{c}} d s A_{c}+\int_{0}^{\left(t-t_{k}\right)} e^{s A_{c}} d s B_{c} K\right) x\left(t_{k}\right)=\Lambda\left(t-t_{k}\right) x\left(t_{k}\right)
\end{aligned}
$$

where $\Lambda(\cdot)$ is given in (3). At the sampling instants $t_{k}$, system (2) is described by the LDI:

$$
x^{+} \in \mathcal{H}(x), \mathcal{H}(x)=\{y: y=\Lambda(\theta) x, \theta \in \mathcal{T}\},
$$

where $x^{+}$represents the state value at $t_{k+1}$, i.e. $x\left(t_{k+1}\right) \in \mathcal{H}\left(x\left(t_{k}\right)\right)$. Consider the initial condition $x_{0} \in \mathbb{R}^{n}$ and a $k$ - length sequence $\sigma=\left\{\theta_{i}\right\}_{i=0}^{k-1} \in S_{k}(\mathcal{T})$. For any $r \in \mathbb{N}, r \leq k$, we denote by $\phi_{\sigma}$ the flow $\left(r, x_{0}\right) \mapsto \phi_{\sigma}\left(r, x_{0}\right)$ defined by $\phi_{\sigma}\left(r, x_{0}\right)=\Phi_{\sigma}(r) x_{0}$ where $\Phi_{\sigma}(r)$ represents the $r$ - step transition matrix of (8) associated to $\sigma$ :

$$
\Phi_{\sigma}(r)= \begin{cases}\Lambda\left(\theta_{r-1}\right) \ldots \Lambda\left(\theta_{1}\right) \Lambda\left(\theta_{0}\right), & r>0 \\ \mathbf{I}, & r=0\end{cases}
$$


The solution of (8) associated to the initial condition $x_{0}$ and to an infinite-length sequence $\sigma \in S_{\infty}(\mathcal{T})$ represents the sequence of points $\left\{\phi_{\sigma}\left(k, x_{0}\right)\right\}_{k=0}^{\infty}$.

Definition 1: The equilibrium point $x=0$ of (8) is said to be globally uniformly exponentially stable if there are constants $c>0,0<\lambda_{d}<1$ s.t.:

$$
\left\|\phi_{\sigma}\left(k, x_{0}\right)\right\| \leq c \lambda_{d}^{k}\left\|x_{0}\right\|, \forall k \geq 0
$$

holds for all initial conditions $x_{0} \in \mathbb{R}^{n}$, all $k \in \mathbb{N}$ and all sequences $\sigma \in S_{\infty}(\mathcal{T})$.

It has been shown in [9] that the stability of the LDI (8) is equivalent to the stability of the continuous-time system (2). The simplest stability criterion is the existence of a quadratic Lyapunov function (a sufficient condition). Note that for LDI, this criterion is not necessary, and may be a conservative stability test [22], [14], [19]. Here we show how to refine the analysis of systems with aperiodic sampling by using Lyapunov functions that are more suitable for LDI.

\section{B. Stability analysis based on discrete-time quasi-quadratic Lyapunov functions}

In what follows, we introduce a new discrete-time stability characterization for systems with aperiodic sampling. Before presenting the approach, we give a technical result for the LDI (8). Several necessary and sufficient conditions exist for the stability of polytopic LDI [17], [15], [14]. However, in the case of sampled-data systems (2), the obtained LDI (8) is not described by a polytopic set of vector fields. It can just be said that the set of matrices described by $\{\Lambda(\theta): \theta \in \mathcal{T}\}$ is a compact subset of $\mathbb{R}^{n \times n}$. As follows, we provide necessary and sufficient stability conditions that underline a converse Lyapunov theorem for LDI defined on compact sets (such as the ones obtained in the case of sampled-data systems).

Theorem 1: Consider the continuous-time system (2) and the equivalent LDI model (8) at the sampling instants. The following statements are equivalent:

1) The equilibrium point $x=0$ of (8) is globally uniformly exponentially stable.

2) There exist a matrix $P=P^{T} \succ 0$ and a positive integer $N$ s.t. the transition matrix $\Phi_{\sigma}(N)$ defined in (9) satisfies the relation

$$
P \succ \Phi_{\sigma}^{T}(N) P \Phi_{\sigma}(N), \forall \sigma \in S_{N}(\mathcal{T})
$$

3) There exists a positive definite function $V: \mathbb{R}^{n} \rightarrow \mathbb{R}^{+}$strictly convex, homogeneous (of the second order), $V(x)=x^{T} \mathcal{L}_{[x]} x$, with $\mathcal{L}_{[\cdot]}: \mathbb{R}^{n} \rightarrow \mathbb{R}^{n \times n}, \mathcal{L}_{[x]}=\mathcal{L}_{[x]}^{T}=\mathcal{L}_{[a x]}, \forall x \neq 0, a \in$ 
$\mathbb{R}, a \neq 0$ s.t. the following relation is satisfied :

$$
V(x)-\max _{\theta \in \mathcal{T}} V(\Lambda(\theta) x)>0 .
$$

Moreover, for $P$ and $N$ satisfying relation (11) in 2), the function $V$ in 3 ) is given by $V(x)=x^{T} \mathcal{L}_{[x]} x$, with $\mathcal{L}_{[x]}=\sum_{i=0}^{N-1} \Phi_{\sigma^{*}(x)}^{T}(i) P \Phi_{\sigma^{*}(x)}(i)$ where

$$
\sigma^{*}(x)=\arg \max _{\sigma \in S_{N-1}(\mathcal{T})} x^{T}\left(\sum_{i=0}^{N-1} \Phi_{\sigma}^{T}(i) P \Phi_{\sigma}(i)\right) x .
$$

The proof is given in the Appendix. The theorem is inspired from [17], [15]. Note that it however goes beyond [17], [15], where only the equivalence 1) $\Leftrightarrow 2$ ) has been established for polytopic LDI. Here we propose, for LDI defined on compact sets, a constructive way of obtaining Lyapunov functions from the inequalities (11). In the generic context of LDI, the theorem can be seen as an alternative to [16] where conditions for the existence of time-dependent Lyapunov functions were derived. The class of functions that we obtain here depends only on the system's state and does not need information on the evolution of the time-varying parameters. The set of inequalities (11) represents a necessary and sufficient stability condition for systems with aperiodic sampling. However, in practice, the number of inequalities to be checked in (11) grows in an exponential manner according to $N$. Therefore, finding a general solution for (11) represents an $N P$-hard ${ }^{1}$ problem [2], even for the simple case when the set $\mathcal{T}$ is finite. Still, one can reduce it to a simpler problem using a finite $N$ and a convexification of the set of vector fields in (8), as follows:

Theorem 2: Consider system (2), the equivalent LDI (8), the set $\mathcal{Z}$ in (4) and the set

$$
\mathcal{Y}(\mathcal{Z})=\left\{Y: Y=\Pi_{i=0}^{N-1} Z_{\mu_{i}}, Z_{\mu_{i}} \in \mathcal{Z}, \mu_{i} \in \mathcal{I}_{p}\right\} .
$$

If there exist a positive integer $N$ and a matrix $P=P^{T} \succ 0$ that satisfy

$$
P \succ Y^{T} P Y, \forall Y \in \mathcal{Y}(\mathcal{Z}), \text { then }
$$

1) the equilibrium point $x=0$ of (8) is globally asymptotically stable;

2) there exists a quasi-quadratic Lyapunov function with the form

$$
V(x)=\max _{i \in \mathcal{I}_{M}} x^{T} L_{i} x
$$

\footnotetext{
${ }^{1}$ There is no numerical algorithm that is able to solve the problem in a polynomial time.
} 
which is strictly decreasing along the solutions of (8), where $L_{i}, i \in \mathcal{I}_{M}, M=p^{N-1}$, are obtained using an enumeration of the elements in the set

$$
\Omega=\left\{Q_{\sigma}^{Z}(N): Q_{\sigma}^{Z}(N)=\sum_{j=1}^{N-1}\left(\Pi_{r=1}^{j} Z_{\mu_{r}}\right)^{T} P\left(\Pi_{r=1}^{j} Z_{\mu_{r}}\right)+P, \sigma=\left\{\mu_{r}\right\}_{r=1}^{N-1} \in S_{N-1}\left(\mathcal{I}_{p}\right)\right\} .
$$

The proof is given in the Appendix. The test involves a finite number of LMI that are sufficient for stability. In Section V, we provide numerical examples where the proposed test is less conservative than the existing ones based on quadratic Lyapunov functions (such as in [8]), or on poly-quadratic stability [13]. Note that the case of quadratic Lyapunov functions is included in the conditions provided here ((11) or (14)) since it corresponds to $N=1$. The accuracy of the stability characterization from conditions (14) mainly depends on two factors: the length $N$ of the horizon of analysis, and the accuracy of the polytopic embedding $\mathcal{Z}$ described in (4) (for more details on such convex embedding see [12], [13], [11]). The amount of conservatism introduced in the approach can be tuned according to these parameters.

\section{COntinuous-Time AnAlysis}

We now provide a characterization of the intersample behavior of (2). Note that (2) is a differential equation with discontinuous right hand side, since $\forall\left(t-t_{k}\right) \in \mathcal{T}$ :

$$
\frac{d x(t)}{d t} \in \Xi\left(t, t_{k}\right), \Xi\left(t, t_{k}\right)=\left\{\left(A_{c}+B_{c} K\right) x(t), A_{c} x(t)+B_{c} K x\left(t_{k}\right)\right\} .
$$

The first element $\left(A_{c}+B_{c} K\right) x(t)$ corresponds to an actuation instant, whereas $A_{c} x(t)+B_{c} K x\left(t_{k}\right)$ corresponds to the intersample dynamics. The model (16) allows for studying the existence of a continuous-time Lyapunov function

$$
V(x)=x^{T} \mathcal{L}_{[x]} x=\max _{i \in \mathcal{I}_{M}} x^{T} L_{i} x,
$$

with $L_{i}, i \in \mathcal{I}_{M}$, a set of symmetric positive definite matrices, as stated in Theorem 3:

Theorem 3: Consider system (2), the equivalent model (16) and the polytopic set $\mathcal{W}$ given in (5). If there exist a scalar $\lambda>0$, a set of scalars $\beta_{i j} \geq 0, i, j=1, \ldots, M$, and matrices $G_{1}, G_{2} \in \mathbb{R}^{n \times n}$ s.t. :

$$
\left(\begin{array}{cc}
A_{c}^{T} L_{i}+L_{i} A_{c}+\lambda L_{i}-\sum_{i \neq j} \beta_{i j}\left(L_{j}-L_{i}\right)+G_{1}+G_{1}^{T} & L_{i} B_{c} K-G_{1} W_{l}+G_{2}^{T} \\
K^{T} B_{c}^{T} L_{i}-W_{l}^{T} G_{1}^{T}+G_{2} & -G_{2} W_{l}-W_{l}^{T} G_{2}^{T}
\end{array}\right) \prec \mathbf{0},
$$


for all $i, j \in \mathcal{I}_{M}$, and for all $l \in \mathcal{I}_{p}$, then the maximal directional derivative of the function (17) following the vector fields of the closed-loop system $U\left(t, t_{k}\right)=\max _{y(t) \in \Xi\left(t, t_{k}\right)} \nabla_{y(t)} V(x(t))$ satisfies the relation

$$
U\left(t, t_{k}\right) \leq-\lambda V(x(t)), \forall t \in\left[t_{k}, t_{k+1}\right], \forall x(t) \neq 0
$$

and the origin $x=0$ of the closed-loop system (2) is exponentially stable.

The proof is given in the Appendix. The goal of the previous theorem is two-fold. First, it can be used for estimating the decay rate of a given quasi-quadratic Lyapunov function (17) over the sampling intervals. If the Lyapunov function is computed from the discrete-time method (Theorem 2), then the $L_{i}$ matrices are given and the computation of the decay rate leads to a set of LMI. Second, the theorem can be applied for directly computing a Lyapunov function without using the discrete-time analysis. The proposed test leads to Bilinear Matrix Inequalities (BMI). However, they can be reduced to LMI by considering quadratic Lyapunov functions, which gives a bit more conservative stability conditions. The previous theorem provides a description of the state behavior at all instants of time (including the intersample behavior): if the set of matrix inequalities (18) are satisfied, then (19) holds, which implies that $V(x(t))<e^{-\lambda t} V\left(x_{0}\right), \forall t>0$. This is the same as $\|x(t)\|^{2}<c e^{-\lambda t}\left\|x_{0}\right\|^{2}$ with $c=\frac{\max _{i \in \mathcal{I}_{M}} \lambda_{\max }\left(L_{i}\right)}{\min _{i \in \mathcal{I}_{M}} \lambda_{\min }\left(L_{i}\right)}$.

Remark 1: Conditions (18) are not feasible in the dead-beat control case, where for some $\theta \in\left[0, \theta_{\max }\right], \Lambda(\theta)$ has eigenvalues at zero. Excluding this case, the existence of functions of the form $V(x)=x^{T} \mathcal{L}_{[x]} x$ is not only sufficient, but also necessary for the stability of (2). This can be easily shown since in this case the matrix $\Lambda(\theta)$ is invertible for all $\theta \in\left[0, \theta_{\max }\right]$. Then $x\left(t_{k}\right)=\Lambda^{-1}\left(t-t_{k}\right) x(t)$ and (2) can be expressed as the linear differential inclusion:

$$
\frac{d x}{d t} \in \mathcal{H}_{c}(x), \mathcal{H}_{c}(x)=\left\{y: y=\left(A_{c}+B_{c} K \Lambda^{-1}(\theta)\right) x, \theta \in\left[0, \theta_{\text {max }}\right]\right\} .
$$

For such differential inclusion, it is known that the existence of a quasi-quadratic Lyapunov function is necessary for stability [19], [14]. However, due to the matrix inversion that appears in (20), no efficient numerical tool exists for applying the results in [14] to (20).

In the following theorem, we restrict to asymptotic stability and propose an analysis method that includes the dead-beat case using the Razumikhin method (see for example [10]).

Theorem 4: Consider system (2) and the polytopic set $\mathcal{W}$ given in (5). If there exist a matrix 
$P=P^{T} \succ 0$, a scalar $\epsilon>0$, and matrices $G_{1}, G_{2} \in \mathbb{R}^{n \times n}$ s.t. :

$$
\left(\begin{array}{cc}
A_{c}^{T} P+P A_{c}+G_{1}+G_{1}^{T}+\epsilon P & P B_{c} K-G_{1} W_{l}+G_{2}^{T} \\
K^{T} B_{c}^{T} P-W_{l}^{T} G_{1}^{T}+G_{2} & -G_{2} W_{l}-W_{l}^{T} G_{2}^{T}-\epsilon P
\end{array}\right) \prec \mathbf{0},
$$

$\forall l \in \mathcal{I}_{p}$, then the origin $x=0$ of the closed loop system (2) is asymptotically stable.

The proof is given in the Appendix. Note that the set of conditions (21) represents an optimization problem which can be solved using a line search algorithm and LMI solvers. The theorem ensures that, within the sampling interval, the Lyapunov-Razumikhin function $V(x)=x^{T} P x$ is always less than the value at the sampling instants, although it is not monotonously decreasing. It can be shown, using numerical examples, that this new approach provides a less conservative stability condition in comparison with the existing continuous-time approaches (see Example 2, in Section V). In fact, this stability test is comparable to the one provided in discrete-time by a common quadratic Lyapunov function. The advantage, w.r.t. the discrete-time approach, is the fact that the intersample behavior is explicitly taken into account and that a sampling interval tending to zero may be considered as well. However, in comparison with Theorem 3, it cannot be used for computing a decay rate over the sampling interval, it only ensures asymptotic stability, not exponential stability.

\section{NumericAl EXAMPLES}

Example 1. Consider a LTI system (2) described by :

$$
A_{c}=\left(\begin{array}{cc}
-0.5 & 0 \\
0 & 3.5
\end{array}\right), B_{c}=\left(\begin{array}{l}
1 \\
1
\end{array}\right) \text { and } K=\left(\begin{array}{ll}
1.02 & -5.62
\end{array}\right) .
$$

$\Lambda(\theta)$ in (3) is Schur for any sampling interval $\theta \in[0,0.46]$. However, switching among different values of $\theta$ in this interval may lead to an unstable behaviour: one can notice that although both $\Lambda(0.25)$ and $\Lambda(0.45)$ are Schur, the transition matrix $\Phi=\Lambda(0.25) \Lambda(0.25) \Lambda(0.45)$ has the eigenvalues outside the unit circle. This implies that when the sampling period is varying in a periodic pattern $0.25 \rightarrow 0.25 \rightarrow 0.45 \rightarrow 0.25 \rightarrow 0.25 \rightarrow 0.45 \ldots$, the closed-loop system is unstable. A similar unstable behavior can be observed for $\theta \in\{0.1,0.43\}$ since the transition matrix $\Phi=(\Lambda(0.1))^{6} \Lambda(0.43)$ is not Schur. We consider that the sampling interval arbitrary switches among the values $\left\{0.1, \theta_{\max }\right\}$ and we use Theorem 2 to compute the maximum $\theta_{\max } \in$ $[0.1,0.46]$ ensuring stability. Using the set of LMI (14), it is possible to find a quasi-quadratic 


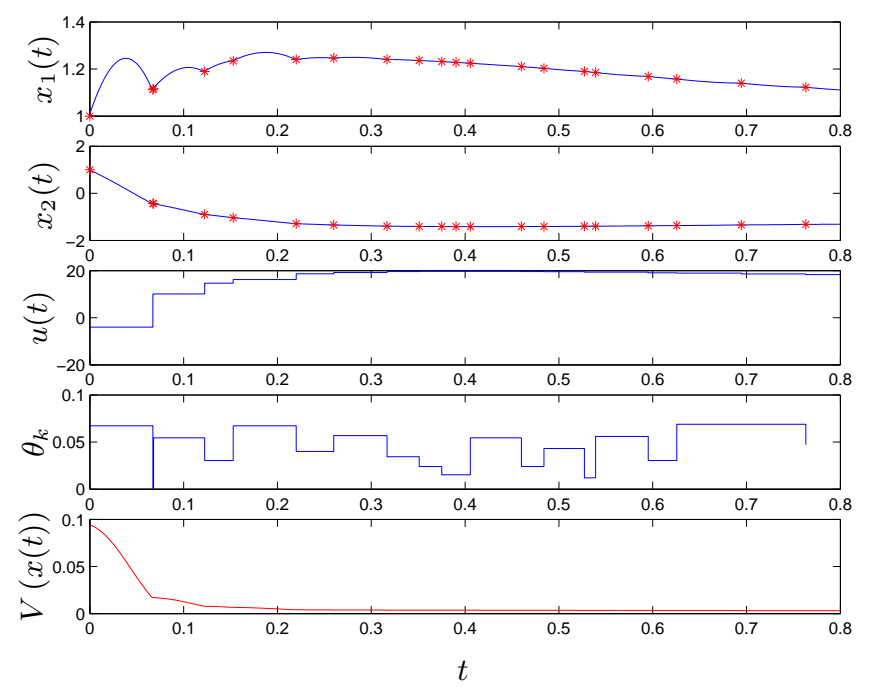

Fig. 1. Simulation for an arbitrary sequence of sampling intervals with $\theta_{\min }=0$ and $\theta_{\max }=0.09$ for the Example 2 .

Lyapunov function of the form (15) for $N=7$ up to $\theta_{\max }=0.41$ (which is very close to the value 0.43 for which an unstable sampling path exists). For $\theta_{\max }=0.41$, using the existing LMI solvers, it is impossible to find a common quadratic Lyapunov function [21], [8], [1] or a polyquadratic one [13]. In fact, the maximum values of $\theta_{\max }$ that can be obtained from quadratic and poly-quadratic Lyapunov functions are $\theta_{\max }=0.36$ and $\theta_{\max }=0.39$, respectively.

Example 2. Consider a continuous-time system described by the following matrices:

$$
A_{c}=\left(\begin{array}{cc}
1 & 15 \\
-15 & 1
\end{array}\right), B_{c}=\left(\begin{array}{l}
1 \\
1
\end{array}\right), K=(5.33-9.33)
$$

In order to construct a polytopic set $\mathcal{W}$ for $\Lambda(\theta)$, we use the method proposed in [13] based on a Taylor series expansion. We use a uniform partition of the interval $\left[0, \theta_{\max }\right]$ into 10 subintervals and apply locally the embedding method (4th order development). Using Theorem 3, a continuous-time quadratic Lyapunov function can be found up to $\theta_{\max }=0.09$ (see Figure 1). For this example the integration operator is singular for $\theta \approx 0.092$ (see Remark 1) which shows that the obtained $\theta_{\max }$ is close to the theoretical bound for quasi-quadratic functions.

The methods in [18], [20], [9] and [6] show that the system is stable for $\theta_{\max }=0.014$, $\theta_{\max }=0.033, \theta_{\max }=0.07$ and $\theta_{\max }=0.12$, respectively. Theorem 4 proves the asymptotic stability for $\theta \in[0,0.14]$ which is less conservative than the existing approaches. Note that using the discrete-time approach (Theorem 2), we are able to show the stability for $\theta \in[0.001,0.15]$. 
This means that Theorem 4 is almost as efficient as the discrete-time approach, with the additional advantage that it takes into account the intersample behavior and very small sampling intervals. Comparing now the number of LMI decision variables, [18] and [9] have $0.5\left(n^{2}+n\right)+m^{2}+m=5$ variables, [20] has $3.5 n^{2}+1.5 n=17$ while [6] has $8 n^{2}+n=34$. In Theorem 4 there are $0.5\left(n^{2}+n\right)+2 n^{2}=11$ variables involved in $p+1=51$ LMI constraints.

\section{CONCLUSIONS}

In this paper we addressed the robust stability of LTI systems with aperiodic sampleddata controller. The approach is based on LMI conditions for the existence of quasi-quadratic Lyapunov functions. It may also be of interest for a more general class of LDI. The intersample behavior is analyzed through a continuous-time analysis that benefits from the discrete-time approach. The results are illustrated by numerical examples that indicate improvement with regard to other recent approaches.

\section{APPENDIX}

Proof of Theorem 1: 1) $\Rightarrow$ 2) Assume that (10) holds. Then there exists a symmetric positive definite matrix $P$ and the associated ellipsoidal norm $\|\cdot\|_{P}$ s.t. $\left\|\phi_{\sigma}(k, x)\right\|_{P}^{2} \leq \frac{\lambda_{\max }(P)}{\lambda_{\min }(P)} c^{2} \lambda_{d}^{2 k}\|x\|_{P}^{2}$ holds for all $x \in \mathbb{R}^{n}$, all $k \in \mathbb{N}$ and all $\sigma \in S_{\infty}(\mathcal{T})$. Since $\lambda_{d}<1$, there exists a positive integer $N$ s.t. $\left\|\phi_{\sigma}(N, x)\right\|_{P}^{2}<\|x\|_{P}^{2}$. This is the same as $\phi_{\sigma}^{T}(N, x) P \phi_{\sigma}(N, x)-x^{T} P x<0, \forall x \in \mathbb{R}^{n}, \forall \sigma \in$ $S_{\infty}(\mathcal{T})$. With $\phi_{\sigma}(N, x)=\Phi_{\sigma}(N) x$, this is the same as $R_{\sigma}(P, N)=\Phi_{\sigma}^{T}(N) P \Phi_{\sigma}(N)-P \prec 0$ $\forall \sigma \in S_{\infty}(\mathcal{T})$. Since $R_{\sigma}(P, N)$ depends only on the first $N$ terms of $\sigma$, then $R_{\sigma}(P, N) \prec 0, \forall \sigma \in$ $S_{N}(\mathcal{T})$, which implies (11).

2) $\Rightarrow$ 3) Consider a sequence $\sigma \in S_{N-1}(\mathcal{T})$. Define

$$
Q_{\sigma}(N)=\sum_{i=0}^{N-1} \Phi_{\sigma}^{T}(i) P \Phi_{\sigma}(i) .
$$

$Q_{\sigma}(N)$ is continuous with respect to $\sigma \in S_{N-1}(\mathcal{T})$. Since $S_{N-1}(\mathcal{T})$ is a compact set, then

$\sup _{\sigma \in S_{N-1}(\mathcal{T})} x^{T} Q_{\sigma}(N) x=\max _{\sigma \in S_{N-1}(\mathcal{T})} x^{T} Q_{\sigma}(N) x$. Thus $V(x)=\max _{\sigma \in S_{N-1}(\mathcal{T})} x^{T} Q_{\sigma}(N) x$ is homogeneous and convex. Furthermore, $P=P^{T} \succ 0$ implies $Q_{\sigma}(N)=Q_{\sigma}^{T}(N) \succ 0$.

We are going to show that if the point 2) is satisfied, then the increment of $V(x)$ over one step satisfies

$$
\Delta V(x)=\max _{\sigma \in S_{N-1}(\mathcal{T})}\left(x^{+}\right)^{T} Q_{\sigma}(N) x^{+}-\max _{\sigma \in S_{N-1}(\mathcal{T})} x^{T} Q_{\sigma}(N) x<0
$$


with $x^{+}=\Lambda(\theta) x$. Consider two $(N-1)$ - length sequences $\alpha=\left\{\alpha_{i}\right\}_{i=0}^{N-2}, \beta=\left\{\beta_{i}\right\}_{i=0}^{N-2} \in$ $S_{N-1}(\mathcal{T})$ defined by $: \alpha=\arg \max _{\sigma \in S_{N-1}(\mathcal{T})}\left(x^{+}\right)^{T} Q_{\sigma}(N) x^{+}, \beta=\arg \max _{\sigma \in S_{N-1}(\mathcal{T})} x^{T} Q_{\sigma}(N) x$. From (23) we obtain

$$
\Delta V(x)=x^{T}\left(\Lambda^{T}(\theta) Q_{\alpha}(N) \Lambda(\theta)-Q_{\beta}(N)\right) x
$$

From the definition of $Q_{\alpha}(N)$, given in (22), with $\sigma=\alpha$, one can notice that

$$
Q_{\alpha}(N)=\sum_{i=0}^{N-1} \Phi_{\alpha}^{T}(i) P \Phi_{\alpha}(i)=\Phi_{\alpha}^{T}(N-1) P \Phi_{\alpha}(N-1)+Q_{\alpha}(N-1) .
$$

We denote by $\gamma$ the sequence $\gamma=\left\{\gamma_{i}\right\}_{i=0}^{N-2} \in S_{N-1}(\mathcal{T})$ with $\gamma_{0}=\theta, \gamma_{i}=\alpha_{i-1}, i=1, \ldots, N-2$. Moreover, we denote by $\delta$ the sequence $\delta=\left\{\delta_{i}\right\}_{i=0}^{N-1} \in S_{N}(\mathcal{T})$, with $\delta_{0}=\theta, \delta_{i}=\alpha_{i-1}, i=$ $1, \ldots, N-1$. One can notice that the term $\Lambda^{T}(\theta) Q_{\alpha}(N) \Lambda(\theta)$ in (24) can be expressed as

$$
\Lambda^{T}(\theta) Q_{\alpha}(N) \Lambda(\theta)=\Phi_{\delta}^{T}(N) P \Phi_{\delta}(N)+Q_{\gamma}(N)-P
$$

Therefore the one-step increment of $V(x)$ is given by

$$
\Delta V(x)=x^{T}\left(\Phi_{\delta}^{T}(N) P \Phi_{\delta}(N)+Q_{\gamma}(N)-P-Q_{\beta}(N)\right) x .
$$

Since $\beta=\arg \max _{\sigma_{2} \in S_{N-1}(\mathcal{T})} x^{T} Q_{\sigma}(N) x$, one can see that $x^{T} Q_{\beta}(N) x \geq x^{T} Q_{\gamma}(N) x$ for any $\gamma \in S_{N-1}(\mathcal{T})$. Therefore $\Delta V(x) \leq x^{T}\left(\Phi_{\delta}^{T}(N) P \Phi_{\delta}(N)-P\right) x$, which means that if the relation (11) is satisfied for all $\delta \in S_{N}(\mathcal{T})$ then condition (12) holds.

3) $\Rightarrow$ 1) Since $V$ is piecewise quadratic and $Q_{\sigma}(N)=Q_{\sigma}^{T}(N) \succ 0$, for all $x \in \mathbb{R}^{n}$ one can find two positive scalars $a, b$ s.t. $a\|x\|^{2}<V(x)<b\|x\|^{2}$ with $a=\inf _{\sigma \in \mathcal{S}_{N}(\mathcal{T})} \lambda_{\min }\left(Q_{\sigma}(N)\right)$ and $b=\sup _{\sigma \in \mathcal{S}_{N}(\mathcal{T})} \lambda_{\max }\left(Q_{\sigma}(N)\right)$. If $V\left(x^{+}\right)-V(x)<0$ for all $x \in \mathbb{R}^{n}$, then there exist a positive scalar $\epsilon<1$ s.t. $V\left(x^{+}\right)<\epsilon V(x)$, which is the same as $V\left(\phi_{\sigma}(k, x)\right)<\epsilon^{k} V(x), \forall x \in \mathbb{R}^{n}, \forall k>$ $0, \forall \sigma \in \mathcal{S}_{\infty}(\mathcal{T})$. This leads to $\left\|\phi_{\sigma}(k, x)\right\|^{2}<\frac{b}{a} \epsilon^{k}\|x\|^{2}$.

Proof of Theorem $2: 1$ ) Since $\Lambda(\theta) \in \mathcal{Z}$, for all $\theta \in \mathcal{T}$, with $\mathcal{Z}$ in (4), then for all sequences of sampling intervals $\sigma \in S_{N}(\mathcal{T})$, the $N$-step transition matrix $\Phi_{\sigma}(N)$ satisfies the relation $\Phi_{\sigma}(N) \in c o(\mathcal{Y})$ with $\mathcal{Y}$ defined in (13). Therefore (14) implies that the conditions (11) in Theorem 1 hold, so the origin of (8) is asymptotically stable.

2) This property can be shown by considering the LDI $x^{+} \in \mathcal{H}^{z}(x), \mathcal{H}^{z}(x)=\left\{Z_{i} x, i \in \mathcal{I}_{p}\right\}$, and showing that (15) is a Lyapunov function for this LDI, using similar arguments to the proof 2) $\Rightarrow$ 3) in Theorem 1. Indeed, one can notice that (14) implies that $V\left(Z_{i} x\right)<V(x), \forall i \in \mathcal{I}_{p}$. 
Since $V(x)$ is convex, $\sum_{i=1}^{p} \alpha_{i} V\left(Z_{i} x\right) \geq V\left(\sum_{i=1}^{p} \alpha_{i} Z_{i} x\right)$, for all positive scalars $\alpha_{i}$, $i \in \mathcal{I}_{p}$ s.t. $\sum_{i=1}^{p} \alpha_{i}=1$. We obtain that $V\left(\sum_{i=1}^{p} \alpha_{i} Z_{i} x\right)<V(x)$. Moreover, since $\Lambda(\theta) \in \mathcal{Z}$, this leads to $V(\Lambda(\theta) x)<V(x)$, i.e. the function (15) is strictly decreasing along the system solutions.

Proof of Theorem 3: Note that $\forall t \in\left[t_{k}, t_{k+1}\right]$, the maximal derivative of the function (17) along the solutions of (2) satisfies the relation : $U\left(t, t_{k}\right) \leq \max _{t-t_{k} \in\left[0, \theta_{\max }\right]} \nabla_{\left(A_{c} x(t)+B_{c} K x\left(t_{k}\right)\right)} V(x(t))$. The value of the maximal directional derivative of $V(x)$ along a vector $y$ is given by:

$$
\nabla_{y} V(x)=\max _{i \in I(x)} 2 x^{T} L_{i} y, I(x):=\left\{i^{*}(x): i^{*}(x)=\arg \max _{i \in \mathcal{I}_{M}} x^{T} L_{i} x\right\} .
$$

Then

$$
U\left(t, t_{k}\right) \leq \max _{t-t_{k} \in\left[0, \theta_{\max }\right]} \max _{i \in I(x)} 2 x(t)^{T} L_{i}\left\{A_{c} x(t)+B_{c} K x\left(t_{k}\right)\right\}
$$

Therefore, condition (19) is satisfied if there exists a $\lambda>0$ s.t.

$$
\max _{t-t_{k} \in\left[0, \theta_{\text {max }}\right]} \max _{i \in I(x)}\left(\begin{array}{c}
x(t) \\
x\left(t_{k}\right)
\end{array}\right)^{T}\left(\begin{array}{cc}
A_{c}^{T} L_{i}+L_{i} A_{c}+\lambda L_{i} & L_{i} B_{c} K \\
K^{T} B_{c}^{T} L_{i} & \mathbf{0}
\end{array}\right)\left(\begin{array}{c}
x(t) \\
x\left(t_{k}\right)
\end{array}\right)<0 .
$$

Equation (7) implies that for $\left(t-t_{k}\right) \in\left[0, \theta_{\max }\right]$, the following relation is satisfied:

$$
\left(\begin{array}{ll}
\mathbf{I} & -\Lambda\left(t-t_{k}\right)
\end{array}\right)\left(\begin{array}{c}
x(t) \\
x\left(t_{k}\right)
\end{array}\right)=\mathbf{0}
$$

Using Finsler's lemma for all $\left(t-t_{k}\right) \in\left[0, \theta_{\max }\right]$ and $i \in \mathcal{I}_{M}$, one can notice that the set of relations

$$
\left(\begin{array}{c}
x(t) \\
x\left(t_{k}\right)
\end{array}\right)^{T}\left(\begin{array}{cc}
A_{c}^{T} L_{i}+L_{i} A_{c}+\lambda L_{i} & L_{i} B_{c} K \\
K^{T} B_{c}^{T} L_{i} & \mathbf{0}
\end{array}\right)\left(\begin{array}{c}
x(t) \\
x\left(t_{k}\right)
\end{array}\right)<0, t-t_{k} \in\left[0, \theta_{\max }\right], i \in \mathcal{I}_{M},
$$

under the constraint (31) is satisfied if there exist matrices $G_{1}, G_{2} \in \mathbb{R}^{n \times n}$ s.t.

$$
\begin{gathered}
\Psi_{i}\left(x(t), x\left(t_{k}\right)\right)=\left(\begin{array}{c}
x(t) \\
x\left(t_{k}\right)
\end{array}\right)^{T}\left(\begin{array}{cc}
A_{c}^{T} L_{i}+L_{i} A_{c}+\lambda L_{i} & L_{i} B_{c} K \\
K^{T} B_{c}^{T} L_{i} & \mathbf{0}
\end{array}\right)\left(\begin{array}{c}
x(t) \\
x\left(t_{k}\right)
\end{array}\right)+ \\
\left(\begin{array}{c}
x(t) \\
x\left(t_{k}\right)
\end{array}\right)^{T}\left\{\left(\begin{array}{c}
G_{1} \\
G_{2}
\end{array}\right)\left(\begin{array}{cc}
\mathbf{I} & -\Lambda\left(t-t_{k}\right)
\end{array}\right)+\left(\begin{array}{c}
\mathbf{I} \\
-\Lambda^{T}\left(t-t_{k}\right)
\end{array}\right)\left(\begin{array}{ll}
G_{1}^{T} & G_{2}^{T}
\end{array}\right)\right\}\left(\begin{array}{c}
x(t) \\
x\left(t_{k}\right)
\end{array}\right)<0(32)
\end{gathered}
$$

for all $\left(x^{T}(t) \quad x^{T}\left(t_{k}\right)\right) \neq \mathbf{0}$, all $\left(t-t_{k}\right) \in\left[0, \theta_{\max }\right]$ and all $i \in \mathcal{I}_{M}$. Therefore, the equation (30) is satisfied if $\max _{i \in I(x)} \Psi_{i}\left(x(t), x\left(t_{k}\right)\right)<0, \forall\left(t-t_{k}\right) \in\left[0, \theta_{\text {max }}\right]$. Using (28), this is the same 
as $\Psi_{i}\left(x(t), x\left(t_{k}\right)\right)<0, \forall i \in \mathcal{I}_{M}, \forall\left(t-t_{k}\right) \in\left[0, \theta_{\max }\right]$, s.t. $x(t)^{T}\left(L_{i}-L_{j}\right) x(t)>0, \forall j \in$ $\mathcal{I}_{M}, j \neq i$. Applying the $\mathrm{S}$-procedure leads to the equation

$$
\left(\begin{array}{cc}
A_{c}^{T} L_{i}+L_{i} A_{c}+\lambda L_{i}-\sum_{i \neq j} \beta_{i j}\left(L_{j}-L_{i}\right)+G_{1}+G_{1}^{T} & L_{i} B_{c} K-G_{1} \Lambda(\theta)+G_{2}^{T} \\
K^{T} B_{c}^{T} L_{i}-\Lambda^{T}(\theta) G_{1}^{T}+G_{2} & -G_{2} \Lambda(\theta)-\Lambda^{T}(\theta) G_{2}^{T}
\end{array}\right) \prec \mathbf{0 .}
$$

Since $\Lambda(\theta) \in \mathcal{W}, \forall \theta \in\left[0, \theta_{\max }\right]$, the previous relation is satisfied when condition (18) holds.

Note that the relation (19) implies that $V(x(t))<e^{-\lambda t} V\left(x_{0}\right) \forall t>0$, which is the same as $\|x(t)\|^{2}<c e^{-\lambda t}\left\|x_{0}\right\|^{2}$ with $c=\frac{\max _{i \in \mathcal{I}_{M}} \lambda_{\max }\left(L_{i}\right)}{\min _{i \in \mathcal{I}_{M}} \lambda_{\min }\left(L_{i}\right)}$.

Proof of Theorem 4: Consider the quadratic function $V(x)=x^{T} P x, P=P^{T} \succ 0$. Define the propositions (A): “ $\dot{V}(x(t))<0$ ", (B): “ $\max _{\theta \in\left[0, \theta_{\max }\right]} V(x(t-\theta))<\alpha V(x(t))$ " and (C): " $V\left(x\left(t_{k}\right)\right)<\alpha V(x(t))$ ". According to the Razumikhin's stability theorem, the trivial solution $x=0$ is asymptotically stable if there exists a scalar $\alpha>1$ and a function $V(x)$ s.t. (B) $\Rightarrow(\mathrm{A})$. Note that $(\mathrm{B}) \Rightarrow(\mathrm{C})$, so it is sufficient to show that there exist a scalar $\alpha>1$ and a matrix $P=P^{T} \succ 0$ s.t. $(\mathrm{C}) \Rightarrow(\mathrm{A})$. The corresponding conditions can be expressed as

$$
\left(\begin{array}{c}
x(t) \\
x\left(t_{k}\right)
\end{array}\right)^{T}\left(\begin{array}{cc}
A_{c}^{T} P+P A_{c} & P B_{c} K \\
K^{T} B_{c}^{T} P & \mathbf{0}
\end{array}\right)\left(\begin{array}{c}
x(t) \\
x\left(t_{k}\right)
\end{array}\right)<0 \text { and }\left(\begin{array}{c}
x(t) \\
x\left(t_{k}\right)
\end{array}\right)^{T}\left(\begin{array}{cc}
-\alpha P & \mathbf{0} \\
\mathbf{0} & P
\end{array}\right)\left(\begin{array}{c}
x(t) \\
x\left(t_{k}\right)
\end{array}\right)<0,
$$

respectively. Using the S-procedure, the stability condition is satisfied if there exists $\epsilon>0$ s.t.

$$
\left(\begin{array}{c}
x(t) \\
x\left(t_{k}\right)
\end{array}\right)^{T}\left(\begin{array}{cc}
A_{c}^{T} P+P A_{c}+\epsilon \alpha P & P B_{c} K \\
K^{T} B_{c}^{T} P & -\epsilon P
\end{array}\right)\left(\begin{array}{c}
x(t) \\
x\left(t_{k}\right)
\end{array}\right)<0
$$

Similarly to the proof of Theorem 3, (31) and the Finsler's lemma, leads to

$$
\left(\begin{array}{cc}
A_{c}^{T} P+P A_{c}+\epsilon \alpha P+G_{1}+G_{1}^{T} & P B_{c} K-G_{1} \Lambda(\theta)+G_{2}^{T} \\
K^{T} B_{c}^{T} P-\Lambda^{T}(\theta) G_{1}^{T}+G_{2} & -G_{2} \Lambda(\theta)-\Lambda^{T}(\theta) G_{2}^{T}-\epsilon P
\end{array}\right) \prec \mathbf{0} .
$$

Since $\Lambda(\theta) \in \mathcal{W}, \forall \theta \in\left[0, \theta_{\max }\right]$, if the set of conditions (21) is satisfied, then there exists a sufficiently small positive $\delta$ s.t. (33) is satisfied with $\alpha=1+\delta$.

\section{REFERENCES}

[1] Andrea Balluchi, Pierpaolo Murrieri, and Alberto L. Sangiovanni-Vincentelli. Controller synthesis on non-uniform and uncertain discretetime domains. In Hybrid Systems: Computation and Control, pages 118-133, 2005.

[2] V. Blondel and J. N. Tsitsiklis. The boundedness of all products of a pair of matrices is undecidable. Systems and Control Letters, 41(2):135 - 140, 2000.

[3] M.B.G. Cloosterman, L. Hetel, N. van de Wouw, W.P.M.H. Heemels, J. Daafouz, and H. Nijmeijer. Controller synthesis for networked control systems. Automatica, 46(10):1584 - 1594, 2001. 
[4] W.P. Dayawansa and C.F. Martin. A converse Lyapunov theorem for a class of dynamical systems which undergo switching. IEEE Transactions on Automatic Control, 44(4), 1999.

[5] F. E. Felicioni and S. J. Junco. A Lie algebraic approach to design of stable feedback control systems with varying sampling rate. In Proceedings of the 17th IFAC World Congress, pages 4881-4886, Seoul, Korea, July 2008.

[6] E. Fridman. A refined input delay approach to sampled-data control. Automatica, 46(2):421 - 427, 2010.

[7] E. Fridman, A. Seuret, and J-P. Richard. Robust sampled-data stabilization of linear systems: an input delay approach. Automatica, 40:1441-1446, 2004.

[8] H. Fujioka. A discrete-time approach to stability analysis of systems with aperiodic sample-and-hold devices. IEEE Transactions on Automatic Control, 54(10):2440-2445, Oct. 2009.

[9] H. Fujioka. Stability analysis of systems with aperiodic sample-and-hold devices. Automatica, 45(3):771 - 775, 2009.

[10] J. Hale and S.M.V. Lunel. Introduction to Functional Differential Equations. Springer, 1993.

[11] M. Heemels, N. van de Wouw, R. Gielen, T. Donkers, L. Hetel, S. Olaru, M. Lazar, J. Daafouz, and S. Niculescu. A comparison of overapproximation methods for stability analysis of networked control systems. In Hybrid Systems : Computation and Control, Lund, Sweden, 2010.

[12] L. Hetel, J. Daafouz, and C. Iung. Stabilization of arbitrary switched linear systems with unknown time varying delays. IEEE Transactions on Automatic Control, 51(10):1668 - 1674, 2006.

[13] L. Hetel, J. Daafouz, and C. Iung. LMI control design for a class of exponential uncertain systems with application to network controlled switched systems. In Proceedings of 2007 IEEE American Control Conference, 2007.

[14] T. Hu and F. Blanchini. Non-conservative matrix inequality conditions for stability/stabilizability of linear differential inclusions. Automatica, 46(1):190 - 196, 2010.

[15] A. Kruszewski, R. Wang, and T-M. Guerra (2008). Non-quadratic stabilization conditions for a class of uncertain non linear discrete-time T-S fuzzy models: a new approach. IEEE Transactions on Automatic Control, 53(2):606-611, 2008.

[16] J.-W. Lee and G. E. Dullerud. Uniform stabilization of discrete-time switched and markovian jump linear systems. Automatica, 42(2):205 - 218, 2006.

[17] A. Megretski. Integral quadratic constraints derived from the set-theoretic analysis of difference inclusions. In Proceedings of the Conference on Decision and Control, Kobe, Japon, 1996.

[18] L. Mirkin. Some remarks on the use of time-varying delay to model sample-and-hold circuits. IEEE Transactions on Automatic Control, 52(6):1109-1112, June 2007.

[19] A. Molchanov and E. Pyatnitskiy. Criteria of asymptotic stability of differential and difference inclusions encountered in control theory. Systems \& Control Letters, 13(1):59-64, 1989.

[20] P. Naghshtabrizi, A. Teel, and J. P. Hespanha. Exponential stability of impulsive systems with application to uncertain sampled-data systems. Systems and Control Letters, 57(5):378 - 385, 2008.

[21] A. Sala. Computer control under time-varying sampling period: An LMI gridding approach. Automatica, 41(12):20772082, 2005.

[22] R. Shorten, F. Wirth, O. Mason, K. Wulff, and C. King. Stability criteria for switched and hybrid systems. Invited paper for SIAM Review, 49(4):545-592, 2007.

[23] W. Zhang. Stability analysis of networked control systems. PhD thesis, Department of Electrical Engineering and Computer Science, CASE Western Reserve University, USA, 2001.

[24] W. Zhang, J. Hu, and A. Abate. On the value functions of the discrete-time switched LQR problem. Automatic Control, IEEE Transactions on, 54(11):2669-2674, Nov. 2009. 